\title{
Nuclear Physics in Stellar Lifestyles with the Trojan Horse Method
}

\author{
Aurora Tumino ${ }^{1,2, *}$, Claudio Spitaleri ${ }^{2,3}$, Marco La Cognata ${ }^{2}$, Silvio Cherubini ${ }^{2,3}$, Giovanni Luca Guardo ${ }^{2}$, Marisa \\ Gulino $^{1,2}$, Iolanda Indelicato ${ }^{2}$, Livio Lamia ${ }^{2,3}$, Rosario Gianluca Pizzone ${ }^{2}$, Giuseppe Gabriele Rapisarda ${ }^{2}$, Stefano \\ Romano $^{2,3}$, Maria Letizia Sergi ${ }^{2}$, and Roberta Spartà ${ }^{2}$ \\ ${ }^{1}$ Facoltà di Ingegneria e Architettura, Università degli Studi di Enna "Kore", Enna, Italy \\ ${ }^{2}$ INFN-Laboratori Nazionali del Sud, Catania, Italy \\ ${ }^{3}$ Dipartimento di Fisica e Astronomia, Università degli Studi di Catania, Catania, Italy
}

\begin{abstract}
The Trojan Horse Method is an indirect technique to measure nuclear reactions of astrophysical relevance at the energies of interest, free of Coulomb suppression and electron screening effects. Its basic features in the framework of the theory of direct reactions will be discussed and the physics case of the ${ }^{12} \mathrm{C}+{ }^{12} \mathrm{C}$ fusion will be addressed.
\end{abstract}

\section{Introduction}

Coulomb repulsion between like charges is a critical issue in nuclear astrophysics, being responsible for the exponential decrease of the cross section $\sigma(E)$ of the relevant nuclear reactions at astrophysical energies. Thus, its behaviour at these low energies is quite often extrapolated from the higher energies by means of the astrophysical $\mathrm{S}(\mathrm{E})$-factor

$$
S(E)=E \sigma(E) \exp (2 \pi \eta)
$$

with $\eta$ the Coulomb parameter of the colliding nuclei, and $\exp (2 \pi \eta)$ the inverse of the Gamow factor that removes the Coulomb dependence of $\sigma(E)$. However, extrapolation can be source of additional uncertainties for $\sigma(E)$ due, for instance, to the presence of unexpected resonances. In the laboratory measurement of nucleosynthesis processes, another critical issue is represented by the electron screening effect that leads to an increased cross section for screened nuclei, $\sigma_{s}(E)$, compared to the cross section for bare nuclei $\sigma_{b}(E)[1,2]$. Therefore, the so called screening factor, defined as

$$
f_{\text {lab }}(E)=\sigma_{s}(E) / \sigma_{b}(E) \approx \exp \left(\pi \eta U_{e} / E\right),
$$

where $U_{e}$ is the so-called "electron screening potential" $[1,2]$, has to be taken into account to determine the bare nucleus cross section. Indeed, this is the key parameter to determine a reaction rate and the only way to get it measured is via indirect methods [3-5] and references therein). They make use of direct reaction mechanisms, such as transfer processes (stripping and pick-up) and quasi-free reactions (knock-out reactions). In particular, the Trojan Horse Method (THM) ([3, 6] and references therein) has been successfully applied many times in the last two decades to reactions connected with fundamental astrophysical problems. Here we present some of the basic ideas of the THM.

*e-mail: tumino@lns.infn.it

\section{Basic ideas of the Trojan Horse Method}

The THM makes use of an appropriate three-body reaction $A+a \rightarrow c+C+s$ performed in quasi free (QF) kinematics at energies well above the Coulomb barrier to extract the cross section of a charged particle two-body process $A+x \rightarrow c+C$ in the Gamow energy window. This is done applying the theory for direct reactions, under the assumption that the nucleus $a$ is described in terms of the $x \oplus s$ cluster structure. In most of the applications performed so far, where $a=$ deuteron, its obvious $p \oplus n$ clusterization was assumed [7-10].

The QF mechanism can be sketched using a pole diagram (see Fig.1) (Shapiro et al. 1968) with two vertexes referring to $a$ break-up (upper vertex) and to the $A+x \rightarrow c+C$ process (lower vertex), with $s$ as spectator. The high energy in the $A+a$ entrance channel ensures that the two body interaction takes place inside the nuclear field, without experiencing either Coulomb suppression or electron screening effects. The $A+a$ relative motion is compensated for by the $x-s$ binding energy, determining the so called "quasi-free two-body energy" given by

$$
E_{Q F}=\frac{m_{x}}{m_{x}+m_{A}} E_{A}-B_{x-s} .
$$

where $E_{A}$ represents the beam energy, $m_{x}$ and $m_{A}$ are the masses of $x$ and $A$ particles respectively, and $B_{x-s}$ is the binding energy for the $x-s$ system. This can be retained as a prescription to determine the best value of the beam energy to populate the relevant $\mathrm{E}_{Q F}$. A cutoff in the momentum distribution reflecting the Fermi motion of $s$ inside the Trojan-horse $a$, defines the range of energies around $\mathrm{E}_{Q F}$ accessible in the astrophysical relevant reaction. In the Plane Wave Approximation, the three body-cross cross section can be factorized as:

$$
\frac{d^{3} \sigma}{d E_{c} d \Omega_{c} d \Omega_{C}} \propto\left[K F\left|\varphi_{a}\left(\mathbf{p}_{s x}\right)\right|^{2}\right]\left(\frac{d \sigma}{d \Omega_{c . m .}}\right)^{\mathrm{HOES}}
$$




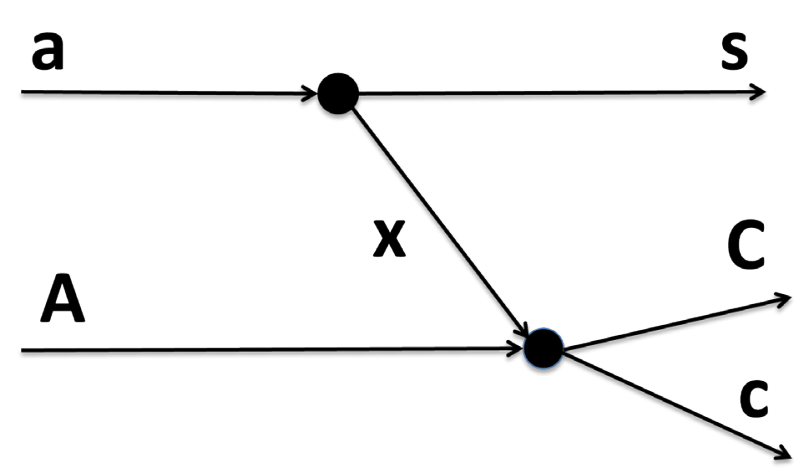

Figure 1. Pole diagram of the $a+A \rightarrow C+c+s$ quasi free reaction.

where KF is a kinematical factor containing the final state phase-space factor. It is a function of the masses, momenta and angles of the outgoing particles [5]; $\varphi_{a}\left(\mathbf{p}_{s x}\right)$ is proportional to the Fourier transform of the radial wave function $\chi(\mathbf{r})$ for the $x-s$ inter-cluster relative motion; $\left(d \sigma / d \Omega_{c . m .}\right)^{\mathrm{HOES}}$ is the half-off-energy-shell (HOES) differential cross section for the binary reaction at the center of mass energy $\mathrm{E}_{c . m}$. given in post-collision prescription by

$$
E_{c m}=E_{c C}-Q_{2 b} .
$$

Here, $Q_{2 b}$ is the $Q$-value of the binary reaction and $\mathrm{E}_{c C}$ is the relative energy of the outgoing particles $c$ and $C$. Using the PWA does not change the energy dependence of the two-body cross section but only its absolute magnitude.

In a typical THM experiment the decay products ( $c$ and $C$ ) of the virtual two-body reaction of interest are detected and identified by means of telescopes (silicon detector or ionization chamber as $\Delta \mathrm{E}$ step and position sensitive detector as E step) placed at the so called quasi free angles. After the selection of the reaction channel, a critical point is to disentangle the quasi free mechanism from other reaction mechanisms feeding the same particles in the final state, e.g. sequential decay and direct break-up. An observable which turns out to be very sensitive to the reaction mechanism is the shape of the experimental momentum distribution of the spectator. In order to reconstruct the experimental $p_{s}$ distribution, the energy sharing method [11] is applied for each pair of coincidence QF angles, selecting $\mathrm{c}-\mathrm{C}$ relative energy windows of 50 to $100 \mathrm{keV}$. The extracted experimental momentum distribution is then compared with the theoretical one and further data analysis is limited to the data lying in the region where the agreement between the two distributions exists (usually within few tens of $\mathrm{MeV} / \mathrm{c}$ ). The theoretical upper limit for the relative momentum $p_{x s}$ between $x$ and $s$ (in the laboratory system $\left.\vec{p}_{x s}=\vec{p}_{x}=-\vec{p}_{s}\right)$ is given by $\kappa_{x s}=\sqrt{2 \mu_{x s} B_{x s}}$ that represents the on-energy-shell (OES) $a=(x s)$ bound state wave number [12].

In the initial idea by [13], the cutoff in $\mathrm{p}_{s}$ was much higher, of hundreds of $\mathrm{MeV} / \mathrm{c}$, because it was needed to compensate for the $A+a$ relative motion. However, this condition is not suitable from the experimental point of view for a number of reasons already pointed out $[3,6]$. Therefore, it is possible to derive the HOES $\left(\left(d \sigma / d \Omega_{c m}\right)^{\mathrm{HOES}}\right.$ from the three-body coincidence yield by simply inverting eq.5. In a final step, the HOES cross section has to be related to the relevant on-energy-shell (OES) cross section by applying the corresponding corrections. In a heuristic approach this consists essentially in replacing the Coulomb suppression in the HOES cross section, by means of the penetrability factor:

$$
P_{l}\left(k_{A x} R\right)=\frac{1}{G_{l}^{2}\left(k_{A x} R\right)+F_{l}^{2}\left(k_{A x} R\right)}
$$

with $F_{l}$ and $G_{l}$ regular and irregular Coulomb wave functions. It was demonstrated that there is no Coulomb barrier in the two-body amplitude extracted from the $\mathrm{TH}$ reaction $[9,14,15]$ and this is due to the virtuality of particle $x$. This seems to be the only consequence of off-energy-shell effects as suggested by the agreement between HOES and OES cross-sections for the ${ }^{6} \mathrm{Li}(\mathrm{n}, \alpha)^{3} \mathrm{H}$ reaction [16]. This procedure does not allow us to extract the absolute value of the two-body cross section. However this is not a real problem since the absolute magnitude can be derived from a scaling to the direct data available at higher energies.

For resonant two-body reactions, $\left(d \sigma / d \Omega_{c . m} .\right)^{\mathrm{HOES}}$ has to be worked out to determine the corresponding on-energyshell (OES) S(E) factor. The corresponding theoretical formalism has been recently developed in a very rigorous way [17] leading to the so called modified R-matrix approach that accounts for HOES effects due to the virtual nature of particle $x$. By fitting the experimental THM cross section, the reduced width amplitude $\gamma$ for entrance and exit channels, energy levels and energy shifts can be deduced and used to determine the astrophysical $S(E)$ factor, since these parameters are the same in both direct and THM data. In this way, an exact parameterization of the astrophysical $S(E)$ factor can be obtained overcoming the extrapolation. If the resonance parameters of a single level in the relevant energy region are known, they can be fixed in the fitting procedure to obtain directly the astrophysical $\mathrm{S}(\mathrm{E})$ factor in absolute units.

Several test studies have been performed in the past years to validate the THM [35], such as the invariance of the two-body reaction amplitude with changing the Trojan Horse nucleus hiding the participant cluster $x$ [19-21], or the use of momentum distributions from Distorted Wave Born Approximation instead of the simple PWA shape, providing same results within experimental errors [42, 44]. The THM has been applied to many reactions of astrophysical interest connected to fundamental problems in different scenarios, from BBN nucleosynthesis [7, 23-40] to AGB and more explosive sites [41-50]. In the last years, reactions involving heavier systems, such as ${ }^{12} \mathrm{C}$ and ${ }^{16} \mathrm{O}$ have been investigated [51, 52].

\section{The ${ }^{12} \mathbf{C}+{ }^{12} \mathbf{C}$ fusion at astrophysical energies}

The ${ }^{12} \mathrm{C}+{ }^{12} \mathrm{C}$ fusion plays a key role in a broad range of scenarios in carbon rich environments. In particular, it determines star evolution and nucleosynthesis of intermediate mass and massive stars $\left(\geq 8 \mathrm{M}_{\odot}\right)$ [53]. It influences also the lower stellar mass limit for carbon ignition. This limit 
separates the progenitors of white dwarfs, novae and type Ia supernovae, from those of core-collapse supernovae, neutron stars, and stellar mass black holes; it constrains superbursts model with neutron and strange stars, in particular if resonances are found to contribute in the Gamow peak [54]; it influences the weak component of the s process, which produces the elements between Fe and Sr. Carbon burning during the hydrostatic phase takes place from 0.8 to $1.2 \mathrm{GK}$, corresponding to center-of-mass energies from 1 to $3 \mathrm{MeV}$. In that sub-Coulomb energy region, the cross section falls rapidly below the nanobarn range. That is why the measurement of the cross section below a center of mass energy $\mathrm{E}_{c m}$ of $2 \mathrm{MeV}$ was never performed. The compound nucleus ${ }^{24} \mathrm{Mg}$ is formed at an excitation energy above the particle decay threshold. Alpha, proton and neutron are the dominant evaporation channels, leading respectively to ${ }^{20} \mathrm{Ne},{ }^{23} \mathrm{Na}$ and ${ }^{23} \mathrm{Mg}$, which can also be produced in excited bound states. Below $2.5 \mathrm{MeV}$ there is not enough energy to feed ${ }^{23} \mathrm{Mg}$ even in its ground state and $\alpha$ and $p$ channel are the only relevant ones at low energies.

The ${ }^{12} \mathrm{C}+{ }^{12} \mathrm{C}$ fusion cross section at the relevant energies was thus determined from the indirect measurement of the ${ }^{12} \mathrm{C}\left({ }^{12} \mathrm{C}, \alpha\right){ }^{20} \mathrm{Ne}$ and ${ }^{12} \mathrm{C}\left({ }^{12} \mathrm{C}, \mathrm{p}\right)^{23} \mathrm{Na}$ reactions via the THM applied to the ${ }^{12} \mathrm{C}\left({ }^{14} \mathrm{~N}, \alpha^{20} \mathrm{Ne}\right)^{2} \mathrm{H}$ and ${ }^{12} \mathrm{C}\left({ }^{14} \mathrm{~N}, \mathrm{p}^{23} \mathrm{Na}\right){ }^{2} \mathrm{H}$ three-body processes in the quasi-free (QF) kinematics regime, where ${ }^{2} \mathrm{H}$ from ${ }^{14} \mathrm{~N}$ is spectator to the ${ }^{12} \mathrm{C}+{ }^{12} \mathrm{C}$ two-body processes [52]. The experiment was performed at the INFN - Laboratori Nazionali del Sud in Catania, Italy. $\mathrm{A}{ }^{14} \mathrm{~N}$ beam accelerated at $30 \mathrm{MeV}$ by the SMP TANDEM was delivered onto a $100 \mu \mathrm{g} / \mathrm{cm}^{2} C$ target with a beam spot on target smaller than $1.5 \mathrm{~mm}$. The experimental setup consisted of two telescopes (38 $\mu \mathrm{m}$ silicon detector as $\Delta \mathrm{E}$ - and $1000 \mu \mathrm{m}$ position sensitive detector (PSD) as E-detector) placed on both sides with respect to the beam direction in symmetric configuration (two on each side), covering angles from $7^{\circ}$ to $30^{\circ}$. The ejectile of the two-body reactions (either $\alpha$ or $p$ ) was detected in coincidence with the spectator $d$ particle. The angular regions covered by the detectors were optimized for the QF kinematics of the break-up process of interest, and the investigated range of deuteron momentum values was feasible to check the existence of the QF mechanism. Several steps are involved in the data analysis (see [52]) and after their completion the two-body cross section of astrophysical relevance was extracted for four channels: ${ }^{20} \mathrm{Ne}+\alpha_{0},{ }^{20} \mathrm{Ne}+\alpha_{1},{ }^{23} \mathrm{Na}+p_{0}$ and ${ }^{23} \mathrm{Na}+p_{1}$. The yield for the ${ }^{20} \mathrm{Ne}+\alpha_{1}$ channel is shown in Fig. 1 (black solid dots) projected onto the ${ }^{12} \mathrm{C}+{ }^{12} \mathrm{C}$ relative energy variable, $\mathrm{E}_{c m}$. A modified one-level many-channel R-matrix analysis was carried out including the ${ }^{24} \mathrm{Mg}$ states reported in [52]. According to the results of [55] at $\mathrm{E}_{c . m .} \leq 3 \mathrm{MeV}$, and monitoring the decrease of the penetration factors for the relevant states, the fraction of the total fusion yield from $\alpha$ and $p$ channels other than $\alpha_{0,1}$ and $p_{0,1}$ was neglected in the modified R-matrix analysis with estimated errors at $\mathrm{E}_{\text {c.m. }}$. below $2 \mathrm{MeV}$ lower than $1 \%$ and $2 \%$ for the $\alpha$ and $p$ channels, respectively. The result for the ${ }^{20} \mathrm{Ne}+\alpha_{1}$ channel is shown in Fig. 1 as middle solid purple line with light purple band arising from the uncertainties on the resonance parame-

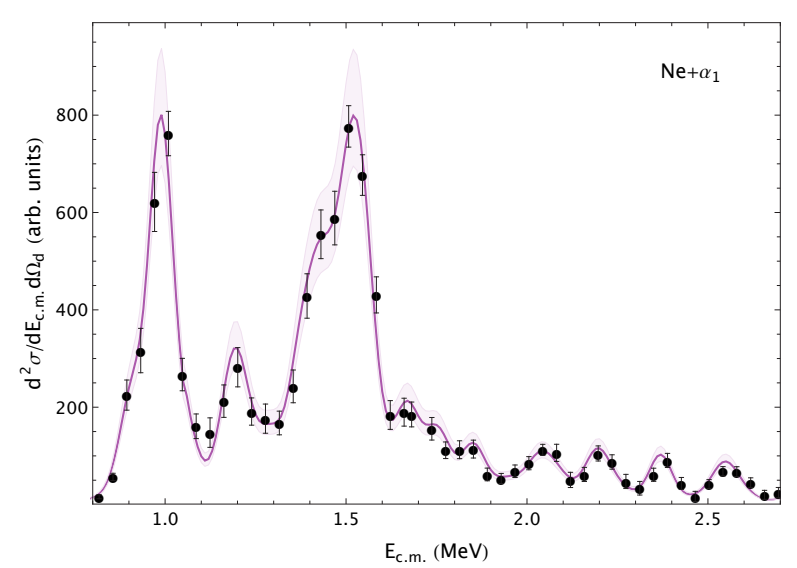

Figure 2. HOES two-body cross section for the ${ }^{20} \mathrm{Ne}+\alpha_{1}$ channel (black filled dots). Solid purple line plus shading from R-matrix calculations including uncertainties of the resonance parameters.

ters, including correlations.

The resonance structure observed in the excitation functions is consistent with ${ }^{24} \mathrm{Mg}$ resonance energies reported in the literature with some tendency for the even $J$ states to be clustered around $1.5 \mathrm{MeV}$ and this might be a sign of intermediate structure of ${ }^{24} \mathrm{Mg}$ associated with a ${ }^{12} \mathrm{C}+{ }^{12} \mathrm{C}$ molecular configuration. The THM reduced widths thus entered a standard R-matrix code and the S(E) factors for the four reaction channels were determined.

The results are shown in Fig. 2 for the ${ }^{20} \mathrm{Ne}+\alpha_{1}$ in terms of modified $\mathrm{S}(\mathrm{E})$ factor, $\mathrm{S}(\mathrm{E})^{*}$, [56]. The black middle line and the grey band represent the best fit curve and the range defined by the total uncertainties, respectively. The grey band is the result of R-matrix calculations with lower and upper values of the resonance parameters provided by their errors.

The resonant structures are superimposed onto a flat nonresonant background taken from [57]. Normalization to direct data was done in the $\mathrm{E}_{\text {c.m. }}$. window $2.5-2.63 \mathrm{MeV}$ of the ${ }^{20} \mathrm{Ne}+\alpha_{1}$ channel where a sharp resonance corresponding to the level of ${ }^{24} \mathrm{Mg}$ at $16.5 \mathrm{MeV}$ shows up and available data [57-60] in this region are the most accurate among those available in the full overlapping region with THM data. The resulting normalization error is $5 \%$. All the existing direct data below $\mathrm{E}_{c . m .}=3 \mathrm{MeV}$ are shown as blue filled circles [57], purple filled squares [58], blue empty diamonds [59], red filled stars [60] and green filled triangles [61]. Except for the data from [57], their low energy limit is fixed by background due to hydrogen contamination in the targets. Disregarding these cases, agreement between THM and direct data is apparent within the experimental errors except for the direct low-energy limit around $2.14 \mathrm{MeV}$, where THM data do not confirm the claim of a strong resonance, rather a nearby one at 2.095 $\mathrm{MeV}$ about one order of magnitude less intense in the ${ }^{20} \mathrm{Ne}+\alpha_{1}$ channel (see Fig. 2) and with similar intensity in the ${ }^{23} \mathrm{Na}+p_{1}$ one. The present result is in agreement with spectroscopy studies of [62, 63] with a deep at 2.14 $\mathrm{MeV}$ and no particularly strong $\alpha$ state around $2.1 \mathrm{MeV}$. Further agreement is found with the unpublished exper- 


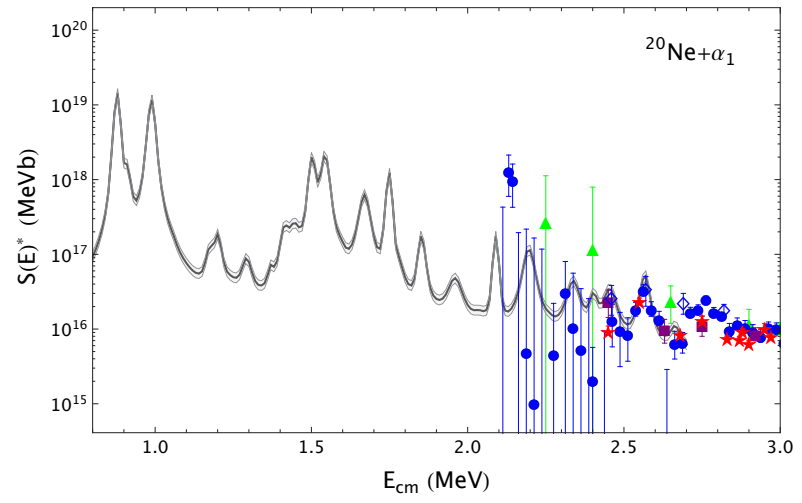

Figure 3. THM astrophysical $\mathrm{S}(\mathrm{E})^{*}$ factor for the ${ }^{20} \mathrm{Ne}+\alpha_{1}$ channel (black solid line). The grey band represents the region spanned by R-matrix calculations with lower and upper values of the resonance parameters. Available direct data in the investigated $\mathrm{E}_{c . m}$. range are reported as purple filled squares [58], blue empty diamonds [59], red filled stars [60], blue filled circles [57] and green filled triangles [61].

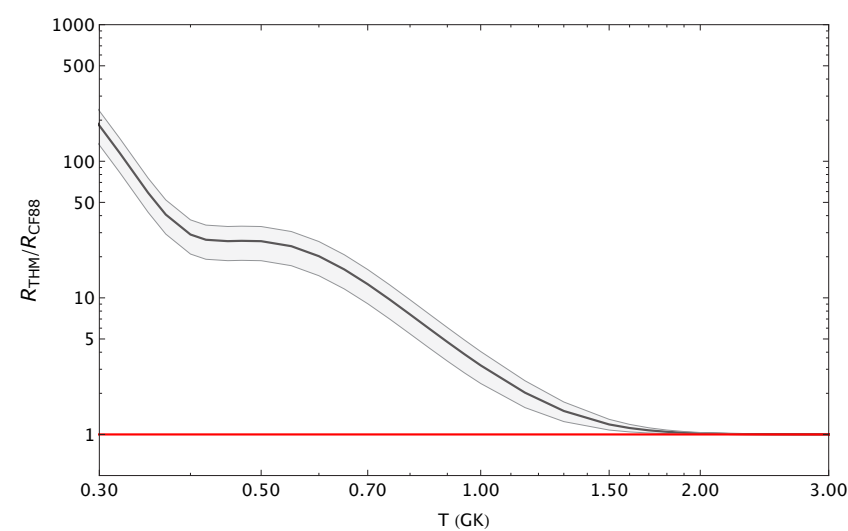

Figure 4. Ratio between the total THM ${ }^{12} \mathrm{C}+{ }^{12} \mathrm{C}$ reaction rate (black line) and the reference one (red line) from [67]. The grey shading defines the region spanned owing to the $\pm 1 \sigma$ uncertainties.

imental data down to $\mathrm{E}_{c . m .}=2.15 \mathrm{MeV}$ from [64] for the ${ }^{12} \mathrm{C}\left({ }^{12} \mathrm{C}, p_{0,1}\right){ }^{23} \mathrm{~N}$ reactions. Our result is also consistent within experimental errors with the total $\mathrm{S}(\mathrm{E})^{*}$ from recent experiments $[65,66]$.

The corresponding reaction rate is shown in Fig. 3 divided by the reference rate from [67]. It experiences a variation below $2 \mathrm{GK}$ with an increase from a factor of 1.18 at $1.2 \mathrm{GK}$ to a factor of more that 25 at $0.5 \mathrm{GK}$. The latter increase, due mainly to the resonant structure around $\mathrm{E}_{c . m .}=1.5 \mathrm{MeV}$, endorses the fiducial value conjectured in [54] to reduce down to a factor of 2 the theoretical superburst ignition depths in accreting neutron stars for a realistic range of crust thermal conductivities and core Urca neutrino emissivities. This change is compatible with the observationally inferred superburst ignition depths. In other words, carbon burning can trigger superbursts. As for the hydrostatic carbon burning regime (0.6 to $1.2 \mathrm{GK}$ ), the present rate change will lower temperatures and den- sities at which ${ }^{12} \mathrm{C}$ ignites in massive post-main-sequence stars. Profiting of the stellar modeling reported in [68], for core C-burning of a star of $25 \mathrm{M}_{\odot}$, the ignition temperature and density would undergo a decrease of down to $10 \%$ and $30 \%$ respectively. Recently, the impact of the new carbon fusion cross sections on Type Ia Supernovae was investigated in [69]. Their progenitors are not well understood. One popular scenario is the double-degenerate (DD) scenario, which attributes SNe Ia to WD-WD binary mergers. The resonance contribution results in a decrease of the carbon burning ignition temperature. Thus, accretion induced collapse occurs more easily and increases the birthrate of Galactic neutron stars with the contribution of the DD scenario to the SNe Ia rate becoming even smaller.

\section{References}

[1] HJ Assenbaum et al., Z. Phys. A 327461 (1987)

[2] F Strieder et al., Naturwissenschaften 88461 (2001)

[3] C Spitaleri et al., Phys. At. Nucl., 74, 1763 (2011)

[4] A Tumino et al., Few Body Syst., 54, Issue 7-10, 869 (2013)

[5] R. Tribble et al., Rep. Prog. Phys. 77, Issue: 10 106901 (2014)

[6] A Tumino et al., Few Body Syst., 54, Issue 5-6 745 (2013)

[7] A Tumino et al., Phys. Rev. C 67065803 (2003)

[8] M La Cognata et al., Eur. Phys. J. A 27249 (2006)

[9] A Tumino et al., Phys. Rev. Lett. 98252502 (2007)

[10] M La Cognata et al., Phys. Rev. C 76065804 (2007)

[11] J Kasagi et al., Nucl. Phys. A 239233 (1975)

[12] I.S. Shapiro, Soviet Phys. Usp., 10(4), 515 (1968)

[13] Baur, G. 1986, Nucl. Phys. A 458, 188 (1986)

[14] AM Mukhamedzhanov et al., Eur. Phys. J. A 27205 (2006)

[15] A Tumino et al., Phys. Rev. C 78064001 (2008)

[16] A Tumino et al., Eur. Phys. J. A 25649 (2005)

[17] AM Mukhamedzhanov, Phys. Rev. C 84044616 (2011)

[18] C Spitaleri et al., Phys. Rev. C 91024612 (2015)

[19] A Tumino et al., Eur. Phys. J. A direct, 1 (2006) DOI: 10.1140/epja/i2006-08-038-1

[20] RG Pizzone et al., Phys. Rev. C 83, 045801 (2011)

[21] RG Pizzone et al., Phys. Rev. C 87, 025805 (2013)

[22] M La Cognata et al., Astrophysical J. Lett. 739, L54 (2011)

[23] RG Pizzone et al., Astronomy \& Astrophysics 398, 423 (2003)

[24] A Tumino et al., Prog. Theor. Phys. Suppl. 154341 (2004)

[25] A Rinollo et al., Nucl. Phys. A 758146 (2005)

[26] Qun-Gang Wen et al., Phys. Rev. C 78035805 (2008)

[27] Qun-Gang Wen et al., J.Phys. G: Nucl. Part. Phys., 38, 085103 (2011)

[28] A Tumino et al., Phys. Lett. B 700, 111 (2011)

[29] A Tumino et al., Phys. Lett. B 705, 546 (2011)

[30] L Lamia et al., Astrophysical J. 768, 65 (2013) 
[31] A Tumino et al., Astrophysical J. 785, 96 (2014)

[32] R.G. Pizzone et al. Astrophysical Journal, 786,112 (2014)

[33] C Spitaleri et al., Phys. Rev. C 90035801 (2014)

[34] Li Chengbo et al., Phys. Rev. C 92, 025805 (2015)

[35] C. Spitaleri et al., Phys. Rev. C 91, 024612 (2015)

[36] L. Lamia Astrophysical J., 850, 175 (2017)

[37] C. Spitaleri et al., Phys. Rev. C 95, 035801 (2017)

[38] Li Chengbo et al., Phys. Rev. C 95, 035804 (2017)

[39] G.G. Rapisarda et al., Eur. Phys. J. A, 54189 (2018)

[40] L. Lamia et al. Astrophysical Journal, 879, 23 (2019)

[41] M La Cognata et al., Phys. Rev. Lett. 101152501 (2008)

[42] ML Sergi et al., Phys.Rev. C R82, 032801 (2012)

[43] M La Cognata et al., Astrophysical J. 708796 (2010)

[44] M. La Cognata et al. Astrophysical J. 739 L54 (2011)

[45] M La Cognata et al., Phys. Rev. Lett. 49, 015106 (2013)

[46] S. Cherubini et al., Phys. Rev. C 92015805 (2015)

[47] R.G. Pizzone et al. Astrophysical Journal, 836, 57 (2017)

[48] I. Indelicato et al. Astrophysical Journal, 845, 19 2017

[49] G. D'Agata et al., Astrophysical J. 860, 61 (2018)
[50] V. Burjan et al., Eur. Phys. J. A 55, 114 (2019)

[51] A. Tumino et al., Eur. Phys. Journ. A, 12327 (2001)

[52] Tumino A. et al., Nature 557, 687 (2018).

[53] Garcia-Berro E. et al., Astrophys. J., 286, 765 (1997).

[54] Cooper R.L. et al., Astrophys. J., 702, 660 (2009).

[55] Becker H.W., Kettner K.U., Rolfs C. \& Trautvetter H.P. Z. Phys. A, 303305 (1981).

[56] Aguilera E.F. et al., Phys. Rev. C 73064501 (2006).

[57] Spillane T. et al., Phys. Rev. Lett. 98, 122501 (2007).

[58] Mazarakis M.G. \& Stephens W.E. Phys. Rev. C, 7 1280 (1973).

[59] M.D. High, B. Cujec, Nucl. Phys. A 282181 (1977)

[60] Kettner K.U., Lorenz-Wirzba H. \& Rolfs C. Z. Phys. A 29865 (1980).

[61] Barrón-Palos L. et al., Nucl. Phys. A 779318 (2006).

[62] Abegg R. \& Davis C.A. Phys. Rev. C 436 (1991).

[63] Caciolli A. et al., NIM B 2661932 (2008).

[64] Zickefoose J. Phys. Rev. C 97065806 (2018).

[65] Jiang C.L. et al., Phys. Rev. C 97 012801(R) (2018).

[66] C. Beck, arXiv:1812.08013

[67] Caughlan G.R. \& Fowler W.A. At. Data Nucl. Data Tables 40, 283 (1988).

[68] Pignatari M. et al., Astrophys. J. 76231 (2013).

[69] Mori K. et al., https://arxiv.org/pdf/1810.01025. 